\title{
METOdOLOGIA DE AVALIAÇÃo DE CARDÁPIO SUSTENTÁVEL PARA SERVIÇOS DE ALIMENTAÇÃO
}

\author{
E. B. F. MOTA, I. W. L. BEZERRA, L. M. J. SEABRA, G. C. B. SILVA, P. M. ROLIM \\ Universidade Federal do Rio Grande do Norte \\ priscillanutri@hotmail.com ${ }^{*}$
}

Submetido 12/2016- Aceito 28/04/2017

DOI: $10.15628 /$ holos.2017.5428

\section{RESUMO}

A elaboração de cardápios saudáveis sob a ótica da produção sustentável é uma tendência mundial entre gestores de serviços de alimentação. O objetivo do estudo foi elaborar uma ferramenta de avaliação para setor de alimentação coletiva, no que diz respeito à produção de refeições e cardápios sustentáveis. Utilizou-se como embasamento científico revisão de literatura nacional e internacional sobre sustentabilidade em serviços de alimentação, e elaborou-se um questionário de verificação. Este questionário foi aplicado em uma UAN institucional e constatou-se conformidade em $70,3 \%$ dos itens avaliados. Destacou-se o desenvolvimento de ações relacionadas a geração de resíduos sólidos e a oferta de variedade das escolhas alimentares, embora, não tenha sido realizada auditoria energética e as refeições não apresentarem adequação total quanto à qualidade nutricional. Diante da relevância e escassez de estudos com propostas de avaliação de cardápios sustentáveis que auxiliem gestores, alunos e professores para aplicação de práticas voltadas para a sustentabilidade, sobretudo visando o atendimento legal vigente, o questionário desenvolvido auxiliará estes profissionais para uma gestão contemporânea, facilitando a implantação de ações sustentáveis na produção de refeições. A relevância do tema e escassez de trabalhos que apresentem ferramentas que propiciem adoção de práticas sustentáveis na produção de refeições ressaltam a importância de mais estudos voltados para esta temática. $O$ instrumento desenvolvido nesta pesquisa é de fácil aplicação e contempla as recomendações mundiais da Associação Americana de Nutricionistas, desta forma, almeja-se que seja utilizado por profissionais dos serviços de alimentação visando adoção de ações sustentáveis na produção de refeições.

PALAVRAS-CHAVE: Cardápios sustentáveis, unidades de alimentação e nutrição, atitudes.

\section{METHODOLOGY OF EVALUATION OF SUSTAINABLE MENU FOR FOOD SERVICES}

\begin{abstract}
The elaboration of healthy menus from sustainable perspective production is a worldwide and imminent trend among professional food service managers. The objective of the study was to elaborate an evaluation tool for collective and commercial food services, in relation to the production of sustainable meals and menus. A review of national and international literature on sustainability in food services was used as a scientific basis. A verification questionnaire was developed. This questionnaire was applied in a collective food service and compliance was verified in $70.3 \%$ of the items. It was highlighted the development of actions such as the audit of solid waste and the variety of food choices, although no energy audit was performed and meals did not present adequate nutritional quality. In view of the relevance and scarcity of studies with proposals for evaluation of sustainable menus that assist the activities
\end{abstract}

of managers of food and nutrition units regarding compliance with actions related to sustainability, above all to comply with current legal aspects, the questionnaire developed will have an impact positive for professionals, fundamental for a contemporary management. The relevance and scarcity of papers that present management tools to adopt more sustainable practices in the production of meals emphasize the importance of more studies focused on this theme. The instrument developed in this research is easy to apply and contemplates the worldwide recommendations of the American Dietetic Association, in this way, it is hoped that it will be used by professionals of the food services aiming the application of sustainable actions in the production of meals.

KEYWORDS: Sustainable menu, food service management, attitudes. 


\section{Introdução}

O setor de Alimentação Coletiva vem sofrendo mudanças ao longo dos anos e encontra-se em constante crescimento no Brasil. Esse setor é representado pelas empresas que produzem refeições para coletividades, em unidades funcionais denominadas Unidades de Alimentação e Nutrição (UAN), as quais são responsáveis pela produção de refeições nutricionalmente balanceadas, com padrão higiênico-sanitário satisfatório, ajustadas financeiramente à realidade econômica da empresa, e numa visão atual e responsável atuando na promoção da saúde e no desenvolvimento de hábitos alimentares saudáveis e sustentáveis (ABERC, 2013; ABREU; SPINELLI; PINTO, 2009; COLARES; FREITAS, 2007; ALVES, 2005).

No contexto atual de sustentabilidade econômica, social, ambiental e de saúde, torna-se imprescindível a oferta de cardápio saudável para consumidores cada vez mais exigentes. Sabe-se que consumo frequente de uma alimentação inadequada pode favorecer o surgimento doenças crônicas, tais como obesidade, diabetes e câncer. $O$ aumento dessas doenças no cenário epidemiológico, ligado às mudanças de estilo de vida da população, como o consumo de alimentos ricos em carboidratos, baixa ingestão de frutas e hortaliças, uso de tabaco e álcool, além da falta de atividade física, é um fator fortemente associado às taxas de morbi-mortalidade e despesas com atenção à saúde no país (GORGULHO; LIPI; MARCHIONI, 2011; (BRASIL, 2010c; TADDEI et al., 2011).

Considerando as evidências científicas convincentes dos benefícios que a alimentação saudável proporciona, sobretudo pelos alimentos que cooperam para a promoção da saúde humana e evitam o aparecimento de doenças crônicas, além do pensamento sustentável, ao garantir que gerações futuras tenham saúde e qualidade de vida, pode-se afirmar que é uma tendência mundial a busca e o incentivo de uma alimentação saudável e, portanto, sustentável (KRAUSE; BAHLS, 2013; BRASIL, 2010b; HARMON; GERALD, 2007; BARRETO et al., 2005).

A implementação de cardápios com a tendência sustentável, ressalta a contribuição primordial do profissional nutricionista inserido em UAN em planejar e supervisionar a produção de refeições, além de distribuir refeições com valor nutricional adequado, adquirir alimentos orgânicos; priorizar fornecedores locais; oferecer variedade de escolhas alimentares, demonstrando preocupação e cuidado com os aspectos sensoriais e culturais da refeição; adequar estruturalmente as unidades e cumprir a lei nacional de Resíduos Sólidos; adquirir produtos químicos biodegradáveis e não tóxicos (ALMEIDA; SANTANA; MENEZES, 2015; ADA, 2001; BRASIL, 2010a; BRASIL, 2010b; VEIROS; PROENÇA, 2010; HARMON; GERALD, 2007; WALLACE, 2005; BRASIL, 2005a; BRASIL, 2005b; CAVALLI, 2001).

A política nacional de resíduos sólidos aborda a gestão de resíduos em uma ordem de prioridade, a saber: não geração, reutilização, reciclagem, tratamento e destinação adequada. Ressalta-se que esta política nacional está em concordância com diretrizes internacionais que tratam a abordagem sustentável em uma hierarquia de prioridades (TATÀNO et al., 2017).

Em termos de gestão de resíduos, os restaurantes ou unidades de alimentação e nutrição representam um desafio e uma oportunidade ao mesmo tempo. $\mathrm{O}$ desafio está relacionado com os esforços necessários para organizar as medidas de gestão de resíduos nestes estabelecimentos; por outro lado, devido a sua expansão e complexidade, os restaurantes podem ser vistos como alvos potenciais para gestão ambiental. Entretanto, têm-se poucas pesquisas técnico-científicas e literatura sobre a gestão de resíduos no setor de alimentação coletiva. Portanto, é necessário aumentar o conhecimento em características qualitativas e quantitativas dos resíduos que podem ser gerados em UAN, bem como as práticas sustentáveis que devem ser adotadas em toda cadeia produtiva (PIRANI e ARAFAT, 2014). Dados da Food and Agriculture Organization (FAO), em documento sobre o estado dos alimentos e agricultura e seus impactos quanto às mudanças 
climáticas e segurança alimentar, demonstram elevados percentuais de perdas e desperdícios de alimentos, sobretudo nas etapas de manuseio e transporte e distribuição dos alimentos (FAO, 2016).

\section{Revisão Bibliográfica}

Conforme proposto pela Organização das Nações Unidas para a Alimentação e a Agricultura (FAO) duas definições sobre sustentabilidade alimentar são importantes: as dietas sustentáveis, as quais são um padrão de alimentação que promove a saúde e o bem-estar e fornece segurança alimentar para a população atual, mantendo recursos humanos e naturais para as gerações futuras; e a segurança alimentar, a qual existe quando todas as pessoas agora, e no futuro, têm acesso a alimentos suficientes, seguros e nutritivos para manter uma vida saudável e ativa. Cardápios sustentáveis são realizados por meio do desenvolvimento de um sistema alimentar que abrange diversos valores que devem ser implantados por meio de práticas e políticas do setor privado e público em toda a cadeia de matérias primas, que vão desde a produção agrícola à distribuição e ao consumo (Figura 1).

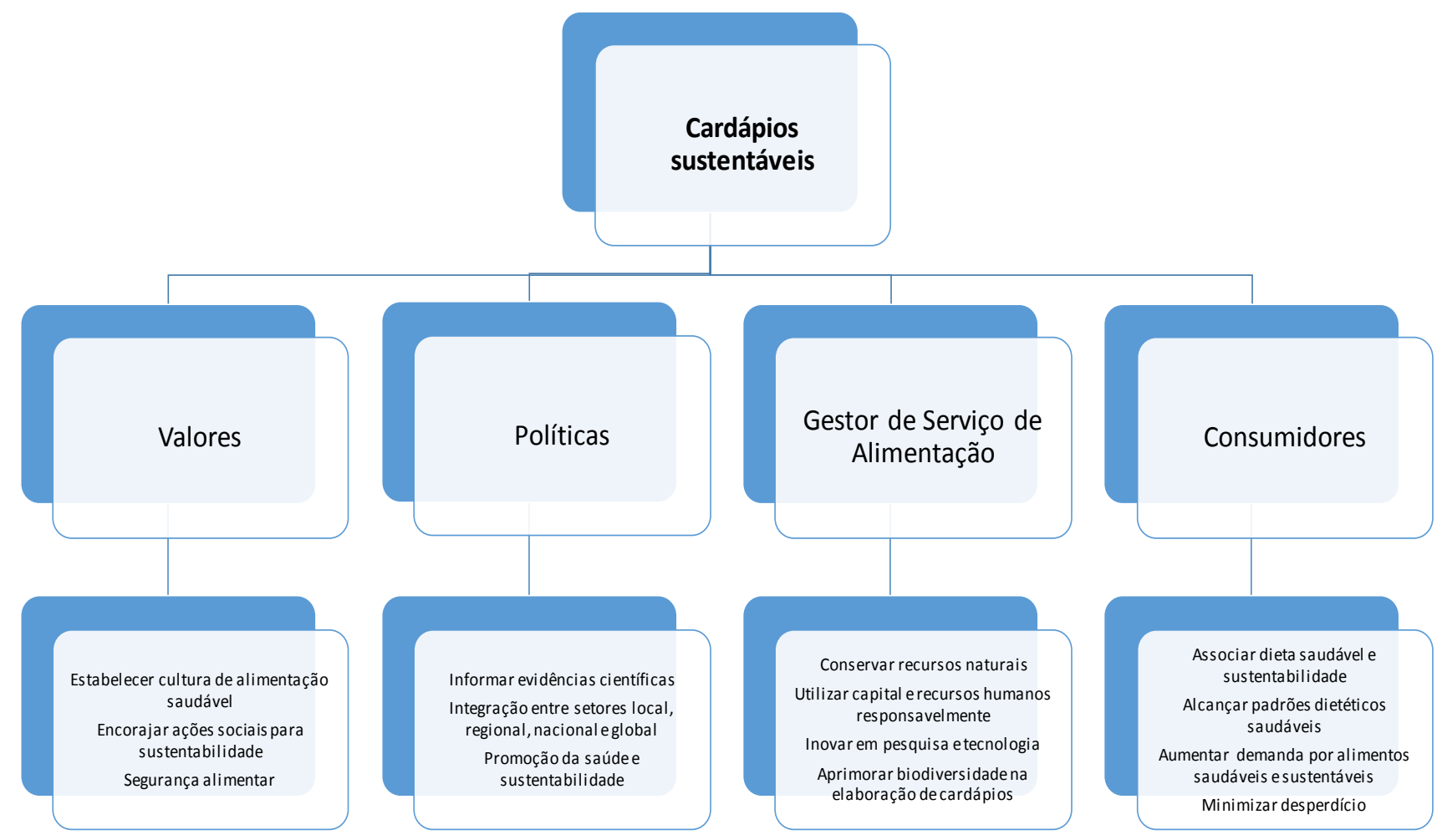

Figura 1. Cardápios sustentáveis numa visão abrangente da sustentabilidade alimentar. Adaptado de Millen et al., 2015.

O impacto ambiental da produção de alimentos é considerável e, se os recursos naturais, como a terra, a água e a energia não forem conservados e geridos de forma otimizada, serão prejudicados e potencialmente perdidos. A produção global de alimentos é responsável por $80 \%$ do desmatamento, mais de $70 \%$ do consumo de água doce e até $30 \%$ das emissões de gases de efeito estufa (GEE) geradas pelo homem. Também é a maior causa de perda de biodiversidade das espécies (HELLER, 2015).

Mudanças climáticas, mudanças nos padrões alimentares da população e demanda por produtos alimentícios, e o crescimento da população continuará a colocar pressões adicionais sobre 
os recursos naturais disponíveis. A satisfação das necessidades alimentares atuais e futuras dependerá, sobretudo, de duas abordagens simultâneas: alterar as escolhas e padrões dietéticos individuais e coletivos e desenvolver práticas agrícolas e de produção que reduzam os impactos ambientais e conservem os recursos, enquanto ainda satisfazem as necessidades alimentares e nutricionais (TILMAN e CLARK, 2014).

Em sistemas de produção de refeições, os alimentos variam amplamente no tipo e quantidade de recursos necessários para a produção, de modo que a demanda dos consumidores que afeta a produção de alimentos também influenciará indiretamente como e em que medida os recursos são usados. Atualmente, o foco das diretrizes dietéticas é mudar os hábitos alimentares dos consumidores para alternativas mais saudáveis e ao mesmo tempo em que são mais protetores dos recursos naturais. Por exemplo, a FAO identificou a dieta mediterrânea como um exemplo de uma dieta sustentável devido à sua ênfase na biodiversidade e porções de carne menores (BURLINGAME e DERNINI, 2011).

Deve-se notar que as pesquisas na área de padrões alimentares e sustentabilidade estão evoluindo rapidamente, além de metodologias para determinar a qualidade dos cardápios quanto aos aspectos nutricionais e sustentáveis. Ações sustentáveis fazem parte dos Objetivos de Desenvolvimento do Milênio e foi conceituada, nos trabalhos da Comissão Mundial da Organização das Nações Unidas (ONU) sobre meio ambiente e desenvolvimento (Comissão de Brundtland), como sendo o "desenvolvimento que satisfaz as necessidades do presente sem comprometer a capacidade das futuras gerações satisfazerem as suas próprias necessidades". Diante disso, percebese que as matérias-primas e processos para transformação e distribuição de alimentos devem ser conservados, portanto, não esgotados ou degradados (BRASIL, 2010d; BARBOSA, 2008).

Veiros e Proença (2010) afirmam que a sustentabilidade é o item central no tripé ambiental, social e econômico. As autoras ressaltam que o desenvolvimento sustentável não está apenas ligado aos aspectos ambientais, como normalmente é a percepção dos profissionais, mas também aos aspectos econômicos e sociais. Entende-se como um grande desafio em promover a sustentabilidade é apropriar-se de informações e lançar o olhar sustentável de modo a fornecer alimentos sob uma ótica mais saudável, e atendendo as demandas ambientais. Esta estratégia de gerenciamento pode fazer a diferença dentro de um mercado extremamente competitivo, como ferramenta educativa, capaz de promover a educação para a sustentabilidade.

De acordo com Barreto et al. (2005), a alimentação saudável também compreende hábitos e práticas alimentares locais, que incentiva o consumo de alimentos regionais e culturais, minimamente processados, economicamente acessíveis e de alto valor nutricional. Acrescenta-se a estes fatores o impacto social e econômico destas ações, já que podem auxiliar na geração de emprego e renda em comunidades agrícolas. Estes aspectos estão diretamente associados à sustentabilidade na produção de refeições.

Neste contexto, o estímulo e o apoio à compra de alimentos produzidos localmente, têm-se mostrado relevantes para o planejamento e implementação de ações dentro de um processo organizacional em um serviço de alimentação. Desta forma, irá fortalecer o setor financeiro e de compras, uma vez que terão iniciativas de articulação para o desenvolvimento de uma gestão sustentável (SARAIVA et al., 2013).

A compra direta de alimentos de produtores locais resulta em diminuição de custos, consumo de alimentos orgânicos, diminuição do êxodo rural, além de estimular o comércio na região, gerando renda para as famílias de pequenos agricultores. Produtos orgânicos são mais saudáveis por conterem menos substâncias tóxicas que podem causar dano à saúde (SOUZA et al., 2012; TRICHES; FROEHLICH; SCHNEIDER, 2011; REAL; SCHNEIDER, 2011). Araújo-Pinto, Peres e Moreira (2012) e Rigotto et al. (2012) mostram em seus estudos alguns efeitos negativos 
potencialmente causados pelo uso de agrotóxico na produção de alimentos, como por exemplo dores de cabeça, alergias, tremores, tonturas, desmaios, doença renal, cânceres, alterações no sistema endócrino e no DNA.

De acordo com Ferment, Fernandes e Avanci (2010), as condições para a segurança alimentar, criadas pela agricultura sustentável, são garantidas por algumas legislações que apresentam os direitos dos agricultores e distinguem caminhos para a proteção da agrobiodiversidade contra o processo de erosão genética. O programa francês "As Boas Refeições da Agricultura Sustentável" nos restaurantes escolares na Seine-Maritime e Haute-Normandie, realizado pela associação Desafios Rurais, faz a seleção dos agricultores por um sistema de aprovação participativa, composto por um regulamento das obrigações e por um diagnóstico de sustentabilidade ampla. Uma das condições obrigatórias para a seleção é a ausência de cultivo de transgênicos nos campos e na alimentação dos animais (ZANONI; FERMENT, 2011).

Outra questão fundamental dentro do contexto da sustentabilidade alimentar é a alimentação orgânica, uma vez que esta possibilita a promoção da segurança alimentar e nutricional e o desenvolvimento sustentável. É de extrema importância a inserção de alimentos orgânicos em diversos segmentos. Há necessidade de atuação integrada para viabilizar a produção orgânica, além disto, na o menos importante, deve-se estimular o consumo desses alimentos pela população em geral (SANTOS et al., 2014).

Como relatam Santos e Santos (2012), a gestão de resíduos em serviços de alimentação ainda é um assunto recente. Embora haja poucas referências na literatura que abordem o tema de planejamento de dietas saudáveis e sustentáveis, são necessários a introdução, a inserção e o aprimoramento da gestão de resíduos sólidos na área de alimentação coletiva. No estudo desses autores, verificou-se que a coleta seletiva não havia sido implantada efetivamente na Unidade de Alimentação e Nutrição selecionada, sendo necessário conscientizar os funcionários sobre proteção ao meio ambiente.

Os resíduos sólidos gerados por organizações que trabalham com alimentos estão concentrados, principalmente, em restos alimentares, com aumento progressivo do desperdício. $\mathrm{Na}$ América, dados ultrapassam valores de $900 \mathrm{Kcal}$ por pessoa/dia em 1974 para $1400 \mathrm{Kcal}$ por pessoa/dia em 2003, e em embalagens, sobretudo de materiais plásticos. A ABNT NBR 10004:2004 também fornece subsídios para o gerenciamento de resíduos sólidos. Essa norma informa que os restos de alimentos gerados por UAN são classificados como não perigosos, embora juntamente com os demais resíduos gerem consequências para o meio ambiente. Assim, a reciclagem destes materiais é, sem dúvida, a melhor alternativa para minimizar problemas ambientais e aumentar o tempo de vida útil dos aterros sanitários (CRUZ et al., 2011; HALL et al., 2009; KINASZ; WERLE, 2008).

Gouveia (2012) destaca a importância da realização do correto gerenciamento desses resíduos, pois inadequações neste sentido geram consequências diretas sobre o ambiente e a saúde, sendo indispensável minimizar as quantidades produzidas por meio da redução, reutilização e reciclagem, já que existem limitações para a destinação final de resíduos.

No real sentido da sustentabilidade, além de fazer aplicar uma gestão integrada de resíduos, desde sua geração até a disposição final, deve-se pensar também sua re-introdução na cadeia produtiva, a fim de conduzir uma adequada gestão, de forma a aperfeiçoar os padrões de produção e consumo, atuando sobre as dimensões da sustentabilidade, entre elas a ambiental, nutricional e de saúde (ZENETI, SÁ E ALMEIDA, 2009).

Silva e Andreoli (2010) sugerem a alternativa da compostagem adequada para resíduos orgânicos, pois é uma solução barata e seu produto final substitui os aditivos químicos e artificiais nas lavouras, atuando como adubo. Destaca-se, ainda, a utilização integral do alimento em 
preparações como uma proposta para diminuição do lixo orgânico originário de serviços de alimentação. É comum ocorrer o descarte de cascas de frutas e de talos de hortaliças, no entanto, essas partes de alimentos contêm nutrientes como proteínas, vitaminas e fibras em quantidades maiores do que as encontradas nas partes normalmente consumidas desses alimentos (KARAM; BARBOZA, 2010; SANTOS; SILVA, 2006). Além disso, existem outras utilidades para cascas de vegetais, como a extração da bromelina da casca do abacaxi, produção do etanol, doces e farinha a partir da casca da banana e utilização da semente do mamão, após secagem, como substitutas da pimenta (MORETTI, 2006).

Neste cenário, existem programas governamentais que incentivam o planejamento e produção de cardápios sustentável, tais como o Cozinha Brasil, realizado pelo Serviço Social da Indústria (SESI), o qual disponibiliza receitas de alimentos para aproveitamento integral dos alimentos (BAHIA, 2015; SESI, 2013) e o Programa Nacional de Alimentação Escolar (PNAE), que determina a presença de gêneros alimentícios utilizados na merenda escolar advindos da Agricultura Familiar e de Empreendedores Familiares Rurais, priorizando, sempre que possível, os alimentos orgânicos e/ou agro ecológicos, in natura, diversificados, sazonais e a oferta de produtos de acordo com o hábito alimentar, preferências e cultura do comensal (BRASIL, 2009; SOUZA et al., 2015).

O tema cardápio sustentável é de grande relevância por se configurar, em âmbito mundial, como uma questão essencial à promoção da saúde e cuidado ambiental, no que diz respeito às diversas praticas sustentáveis que podem ser aplicadas desde o planejamento à distribuição de refeições em serviços de alimentação. Diante da relevância e escassez de metodologias de avaliação de práticas sustentáveis em UAN, este estudo propõe uma ferramenta de trabalho para nutricionistas de UAN no que diz respeito à produção e à distribuição de cardápios saudáveis e sustentáveis.

\section{Metodologia}

O estudo foi do tipo descritivo, transversal e de natureza quali e quantitativa, realizado em uma Unidade de Alimentação e Nutrição que oferece cardápio do tipo médio, com distribuição tipo self-service com porcionamento de um prato protéico. Quanto aos aspectos éticos, este trabalho foi aprovado pelo Comitê de Ética em Pesquisa do Hospital Universitário Onofre Lopes (HUOL-UFRN) sob protocolo de número 81179.

A proposta metodológica se baseou na elaboração e aplicação de um questionário, visando à identificação de aspectos recomendados pela American Dietetic Association (ADA) (2001), Veiros e Proença (2010), Lei 12.305 de 2010 (BRASIL, 2010a), Wallace (2005), Harmon e Gerald (2007), Brasil Food Trends 2020 (BRASIL, 2010b), Cavalli (2001) e pelo Guia Alimentar para a população brasileira (BRASIL, 2014) quanto à saudabilidade e sustentabilidade de cardápios em UAN.

Os itens do questionário foram preenchidos da seguinte forma: em conformidade (S), quando recebia resposta positiva, não conformidade $(\mathrm{N})$, quando recebia resposta negativa, ou Não se Aplica (NA). Para os questionamentos que possuíam subitem, todos deveriam receber respostas positivas para que o item fosse considerado em conformidade. Os resultados foram obtidos de acordo com a porcentagem de itens $\mathrm{S}, \mathrm{N}$ e NA.

$O$ instrumento foi aplicado por 3 vezes pelo mesmo pesquisador, em dias diferentes, com intervalo de uma semana entre as aplicações, e posteriormente foi realizada uma média das respostas obtidas. Alguns requisitos do instrumento foram respondidos por meio de observação do pesquisador durante os dias de coleta dos dados. 


\section{Resultados e Discussão}

Os resultados obtidos com a aplicação do instrumento para avaliar ações sustentáveis na produção de refeições demonstraram que a UAN avaliada apresentou $70,3 \%$ de cumprimento de práticas voltadas para a abordagem sustentável. O quadro 1 apresenta o instrumento de avaliação proposto.

\section{Questionário de avaliação sobre os aspectos de sustentabilidade no planejamento e distribuição de cardápios para Unidades de Alimentação e Nutrição (UAN)}

\begin{tabular}{|c|c|c|c|}
\hline Itens avaliados & S & N & NA \\
\hline
\end{tabular}

O gestor realiza compra de alimentos direto de produtores locais?

$O$ gestor da UAN adquire produtos orgânicos?

O gestor reduz a dependência dos alimentos importados e ultraprocessados?

O gestor utiliza critérios adequados quanto aos aspectos nutricionais para substituição de preparações do cardápio em caso de situações imprevistas?

0 gestor oferece variedade de escolhas alimentares?

O gestor inclui a seleção de preparações regionais e o resgate do patrimônio gastronômico no cardápio?

O gestor apresenta cuidado com os aspectos sensoriais da refeição?

O gestor define e executa adequadamente as técnicas de preparo?

O gestor distribui cuidadosamente as preparações para estimular principalmente o consumo de alimentos vegetais, tais como:

( )Frutas ( )Verduras ( )Legumes ( )Grãos integrais?

A UAN possui local de consumo da refeição adequado durante todo o período de distribuição?

Os bens e serviços disponibilizados pela UAN são devidamente qualificados e adequados às atividades?

O gestor utiliza ingredientes ou matérias primas com ingredientes transgênicos em sua composição na produção de refeições?

O gestor distribui preparações seguras que utilizam cascas e talos de vegetais como ingredientes?

O gestor aplica a lei 12.305/2010, sobre a Política Nacional de Resíduos Sólidos? ( )Realiza coleta seletiva ( )Recicla ou disponibiliza materiais para reciclagem ( )Minimiza o desperdício de alimentos ( )Reutiliza materiais ( ) Recicla ou disponibiliza o óleo de cozinha utilizado nas fritadeiras para reciclagem ( )Doa restos de comida para a compostagem ou alimentação animal ( )Adquire materiais reciclados ( )Adquire materiais com embalagens que podem ser recicladas e/ou reutilizadas ( )Incentiva a indústria de reciclagem

Os produtos utilizados na UAN possuem origem comprovada, não sendo produzidos por menores de idade ou trabalho escravo?

Há controle de ruído na UAN?

O consumo energético e hídrico da UAN são suficientes?

$O$ gestor realiza controle de perdas e sobras de alimentos?

O gestor utiliza alguma forma de energia renovável?

HOLOS, Ano 33, Vol. 04 


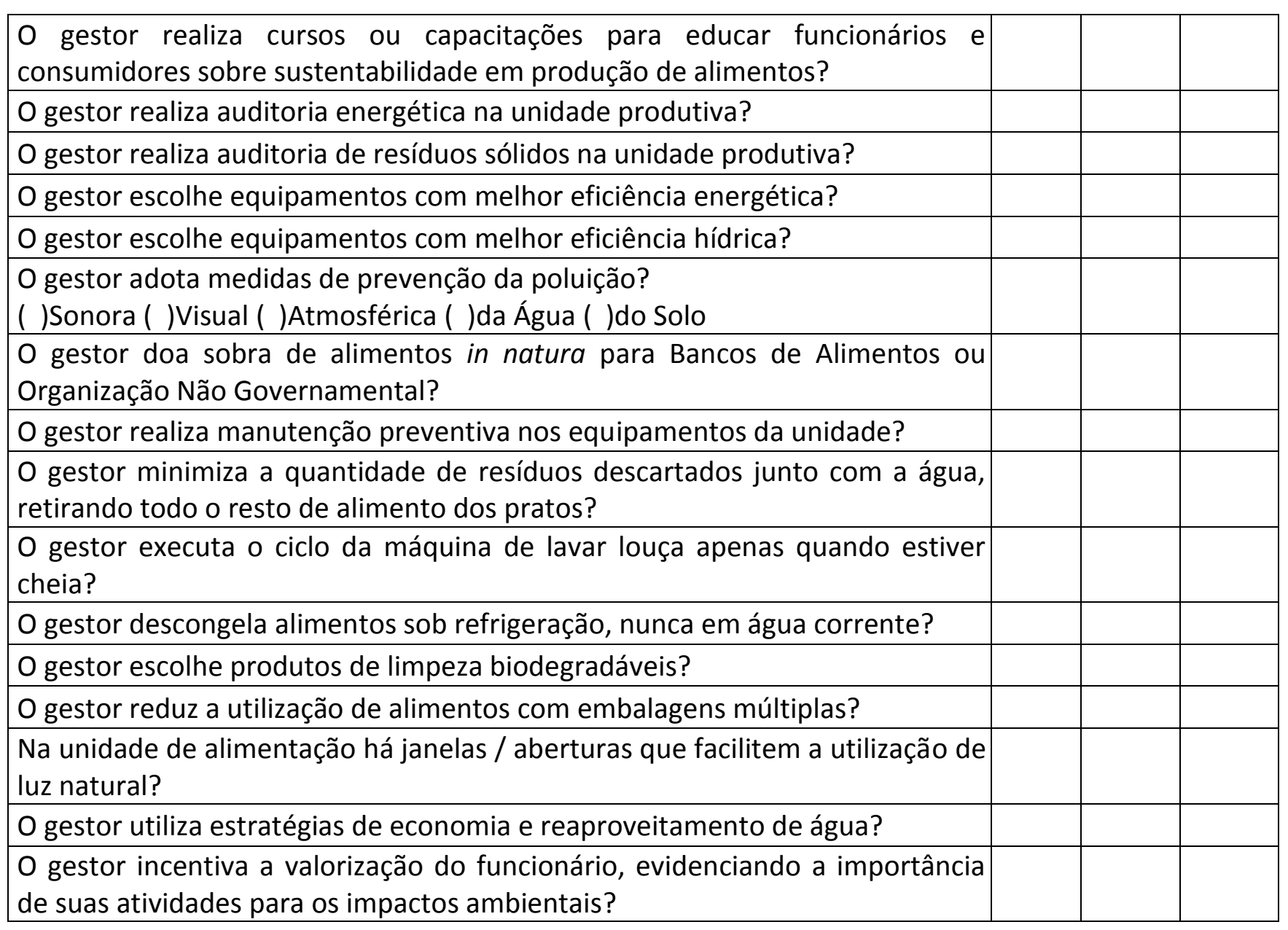

Quadro 1. Instrumento de avaliação de ações sustentáveis na produção de refeições.

$\mathrm{S}=\operatorname{Sim}$ (conforme); $\mathrm{N}=$ Não (não-conforme); NA = Não se aplica. Fontes: ADA (2001); Veiros e Proença (2010); Lei 12.305/2010; Wallace (2005); Harmon e Gerald (2007); BRASIL (2010b); BRASIL (2005a) e Cavalli (2001).

A utilização do instrumento na prática profissional do gestor de UAN pode ser considerada simples, de baixo custo, no entanto representa relevância e subsídios para intervenções em diversas atividades dos serviços de alimentação. Na UAN em que o questionário foi aplicado, 70,3\% das questões sobre sustentabilidade na produção de refeições encontraram-se em conformidade com as literaturas científicas da área de sustentabilidade, o que certamente representa um bom indicativo de cumprimento de ações sustentáveis.

As respostas evidenciaram que na UAN há redução da dependência de alimentos importados; auditoria de resíduos sólidos, incluindo aplicação da lei nacional 12.305 de 2010 (BRASIL, 2010a); escolha de equipamentos com melhor eficiência energética; adoção de medidas de prevenção de diferentes tipos de poluição; doação de sobras de alimentos in natura para Bancos de Alimentos ou ONGs; realização de manutenção preventiva nos equipamentos; produtos e matérias primas adquiridos com origem comprovada, não sendo produzidos por menores de idade ou trabalho escravo; controle de ruído; consumo energético e hídrico suficientes; controle de perdas/sobras; energia renovável; minimização da quantidade de resíduos descartados junto com a água; oferta de variedade de escolhas alimentares, incluindo a seleção de preparações regionais e o resgate do patrimônio gastronômico; janelas que facilitam a entrada de luz natural; estratégias de economia e reaproveitamento de água de cozimento; valorização do funcionário; distribuição de 
preparações seguras que utilizam cascas de vegetais como ingredientes; e produtos de limpeza biodegradáveis, como o detergente e o sabão em pó.

O estudo de Barthichoto et al. (2013) também avaliou práticas de sustentabilidade em UAN, entretanto identificou que a maior parte dos locais entrevistados não seguiam medidas de produção sustentável, sobretudo com relação à seleção e adequada destinação de resíduos, além da não utilização de forma prioritária embalagens recicláveis, ausência de controle de sobras de alimentos e ficha técnica de preparação.

Martins (2015) analisou as práticas de sustentabilidade ambiental adotadas por UAN coletivas em Santa Catarina e considerou como práticas sustentáveis importantes a utilização de alimentos regionais, uso de lâmpadas energeticamente eficientes, realização de coleta seletiva, atenção a sazonalidade dos alimentos, descongelamento de alimentos sem uso de água corrente e o destino adequado de óleo de fritura.

O serviço de alimentação avaliado no presente estudo pelo instrumento atendeu grande parte dos aspectos necessários para execução de cardápios sustentáveis. Entretanto, os gestores responsáveis devem implementar requisitos como a compra direta de alimentos de produtores locais; aquisição de produtos orgânicos; oferecer cursos que visem educar funcionários e comensais sobre a sustentabilidade em produção de alimentos; reduzir a utilização de alimentos com embalagens múltiplas; distribuição refeições com melhor qualidade nutricional, diminuindo o teor de sódio, proteínas, colesterol e calorias nas refeições; e a não utilização de matérias-primas com ingredientes transgênicos em sua composição para a produção de refeições.

A qualidade nutricional de um cardápio é um parâmetro importante na avaliação de um serviço de alimentação, pois interfere diretamente na saúde dos usuários. No entanto, percebe-se que a unidade estudada distribui refeições com aporte calórico e nutricional inadequados, portanto, neste ponto, não cumpre com as recomendações de um cardápio sustentável. Este fato corrobora com outro estudo em que se visualizaram inadequações na qualidade e gestão de cardápios (GERALDO, BANDONI E JAIME, 2008).

Para parcela dos trabalhadores que freqüentam a organização estudada na pesquisa, os alimentos consumidos no seu local de trabalho podem representar a refeição mais importante do seu dia. Então, para seguir os princípios da sustentabilidade, que não está ligado apenas aos aspectos ambientais, quando o cardápio ofertado cumprir todas as recomendações para uma alimentação saudável, papel desempenhado e de responsabilidade do profissional em nutrição, proporcionará produtividade no trabalho, diminuição do absenteísmo, aumento da qualidade de vida e prevenção de doenças crônicas, o que conseqüentemente evitará gastos futuros de despesas com a saúde (BRASIL, 2005b; VEIROS E PROENÇA, 2010; HARMON; GERALD, 2007; VEIROS et al., 2006; WANJEK, 2005).

O serviço de alimentação avaliado no presente estudo não realiza compra direta de alimentos de produtores locais. Recomenda-se que haja intervenção para esse aspecto, pois, como descreve O'Kane (2011), os sistemas alimentares da comunidade, juntamente com os profissionais envolvidos na gestão de produção de refeições, podem desempenhar um papel importante para conter o crescimento dos níveis de obesidade da atualidade, através da utilização de métodos de produção mais sustentáveis de alimentos, o desenvolvimento das economias locais e permitindo conexões mais próximas entre agricultores e consumidores.

No estudo feito por Lima (2012) concluiu-se que os comensais de restaurantes colocam a presença de alimentos orgânicos como sendo um dos fatores mais importantes para se caracterizar um cardápio sustentável. É movido por essa ideia que cresce o consumo por esse tipo de alimento (LIMA et al., 2015). 
Destaca-se, como resultado desta pesquisa, a utilização de produtos transgênicos na produção de refeições. Cavalli (2001) comenta que o consumo de produtos transgênicos fere a segurança alimentar, portanto os princípios da sustentabilidade, pois há uma série de riscos para a saúde, atualmente questionados e alvos de inúmeras pesquisas para o conhecimento mais aprofundado da relação do consumo com o aumento das alergias, resistência a antibióticos, aumento das substâncias tóxicas e de resíduos nos alimentos. Por outro lado, pode-se perceber que no presente estudo houve conformidade quanto a este quesito, pois os gestores responsáveis realizam auditoria de resíduos sólidos, aplicando a lei 12.305 de 2010, devendo, inclusive, servir de exemplo para UAN que ainda não aderiram a tal recomendação.

Por último, foi verificado que a edificação da unidade de alimentação avaliada não foi realizada coerente com um processo sustentável. Essa visão de considerar o meio ambiente já está presente na construção civil internacional, sendo concedidos às empresas incentivos fiscais. As "edificações sustentáveis" praticam, durante todas as etapas da construção e utilização, o uso racional de recursos naturais, utilizam materiais ecologicamente corretos, privilegiam materiais naturais e/ou recicláveis em geral, e altera o mínimo possível o ambiente no qual estão inseridas, além de promover o bem estar social e aumento da produtividade dos usuários. Sugere-se que a unidade ponha em prática essa tendência em casos de reformas e/ou ampliações de sua estrutura física (LAMBERTS et al., 2008).

Novos desafios vêm sendo impostos aos diversos segmentos de organizações, no sentido de atingirem e demonstrarem desempenhos ambientais, econômicos e sociais adequados, controlando os impactos de suas relações, processos, produtos e serviços na sociedade, de forma consistente com sua política e seus objetivos de responsabilidade social (SHCOLNIK, 2011).

\section{Conclusão}

O instrumento proposto se mostrou viável para avaliar a unidade no que se refere sobretudo ao planejamento adequado de cardápios como potencial ferramenta para garantir sustentabilidade em serviços produtores de refeições. A unidade avaliada demonstrou a implementação de ações que buscam otimizar a produção de refeições numa abordagem sustentável, porém são necessárias a melhoria no que diz respeito a oferta de um cardápio equilibrado, adequado em calorias e nutrientes, e sensorialmente atrativo para clientela, bem como a realização de auditoria energética e de resíduos sólidos. Desta forma, a Unidade cumprirá com os princípios da sustentabilidade: econômico, ambiental e social, tendo em vista que o cardápio oferecido contribuirá com a saúde da clientela, evitando despesas futuras, manutenção dos recursos naturais e respeito ao cliente.

O instrumento de avaliação proposto é extremamente importante para verificação do cumprimento das ações relacionadas com a saudabilidade e sustentabilidade em organizações produtoras de refeições, além de fácil aplicação. Mais estudos devem ser incentivados para avaliação da gestão de cardápios sustentáveis, levando-se em consideração o cenário contemporâneo 


\section{Referências}

ABERC. Associação Brasileira de Empresas de Refeições Coletivas. História e mercado. Disponível em: <http://www.aberc.com.br/mercadoreal.asp?IDMenu=21> Acesso em 16 de maio de 2013.

ABNT. Associação Brasileira de Normas Técnicas. NBR 10004: Resíduos Sólidos: Classificação. Rio de Janeiro, 2004.

ABREU, E. S., SPINELLI, M. G. N., PINTO, A. M. S. Gestão de unidades de alimentação e nutrição: um modo de fazer. 3.ed. [S. I.]: Editora Metha, 2009.

ADA. (2001). Position of the American Dietetic Association: dietetics professionals can implement practices to conserve natural resources and protect the environment. Journal of the American Dietetic Association, v. 101, n.10, p. 1221-1227.

ALMEIDA, J. L., SANTANA, K. B., MENEZES, M. B. C. Sustentabilidade em unidades de alimentação e nutrição. 8o encontro internacional de formação de professores, v.8 n.1, 2015.

Alves, F. S. A organização da produção de unidades de alimentação e nutrição. 2005. 158 f. Dissertação (Mestrado em Administração) - Programa de Pós-graduação em Administração, Universidade Federal de Santa Catarina, Florianópolis, 2005.

Araújo-Pinto, M., Peres, F., Moreira, J. C. (2012). Utilização do modelo FPEEEA (OMS) para a análise dos riscos relacionados ao uso de agrotóxicos em atividades agrícolas do estado do Rio de Janeiro. Ciência e Saúde Coletiva, v.17, n.6, p.1543-1555.

BAHIA, G. D. Programa social cozinha Brasil e a contribuição para o desenvolvimento sustentável. Trabalho de conclusão de curso apresentado ao IDP - Instituto Brasiliense de Direito Público, curso de Pós-Graduação lato sensu, nível de Especialização em Direito da Propriedade, Agronegócio e Desenvolvimento Sustentável, como pré-requisito para a obtenção do título de Especialista. Brasília, 2015.

Barreto, S. M., Pinheiro, A. R. O., Sishieri, R., Monteiro, C. A., Batista Filho, M., Schimidt, M. I., Lotufo, P., Assis, A. M., Guimarães, V., Recine, E. G. I. G., Victora, C. G., Coitinho, D., Passos, V. M. (2005). A. Análise da Estratégia Global para Alimentação, Atividade Física e Saúde, da Organização Mundial da Saúde. Epidemiologia e Serviços de Saúde, v.14, n.1, p. 41 - 68.

Barthichoto, M., Matias, A. C. G., Spinelli, M. G. N., Abreu, E. S. (2013). Responsabilidade ambiental: perfil das práticas de sustentabilidade desenvolvidas em unidades produtoras de refeições do bairro de higienópolis, município de São Paulo. Qualit@s Revista Eletrônica, v.14, n.1, p.1-12.

BRASIL. Ministério da Saúde. Inquérito Domiciliar sobre Comportamentos de Risco e Morbidade Referida de Doenças e Agravos não Transmissíveis: 15 capitais e Distrito Federal 2002-2003. Rio de Janeiro: INCA, 2004.

Burlingame, B., Dernini, S. (2011). Sustainable diets: the Mediterranean diet as an example. Public Health Nutrition, v.14, n.12A, 2285-7.

Brasil. Ministério da Saúde. Secretaria de Atenção à Saúde. Departamento de Atenção Básica. Guia alimentar para a população brasileira / Ministério da Saúde, Secretaria de Atenção à Saúde, Departamento de Atenção Básica. - 2. ed. - Brasília : Ministério da Saúde, 2014. 156 p.

Conselho Federal de Nutricionistas. Resolução no 380/2005. Dispõe sobre a definição das áreas de atuação do nutricionista e suas atribuições, estabelece parâmetros numéricos de referência, por área de atuação, e dá outras providências. Brasília, 2005b.

Ministério da Educação. Resolução no 38, de 16 de julho de 2009. Dispõe sobre o atendimento da alimentação escolar aos alunos da educação básica no Programa Nacional de Alimentação Escolar - PNAE, Brasília, DF, 16 jul. 2009.

Presidência da República. Casa Civil. Subchefia para Assuntos Jurídicos. Lei no 12.305, de 2 de agosto de 2010. Política Nacional de Resíduos Sólidos, 2010a. 
Federação das Indústrias do Estado de São Paulo. Instituto de Tecnologias de Alimentos. Brasil Food Trends 2020. São Paulo, 2010b.

CAVALLI, S. B. (2001). Segurança alimentar: a abordagem dos alimentos transgênicos. Revista de Nutrição, Campinas, v.14, p.41-46.

Colares, L. G. T., Freitas, C. M. (2007). Processo de trabalho e saúde de trabalhadores de uma unidade de alimentação e nutrição: entre a prescrição e o real do trabalho. Cadernos de Saúde Pública, v.23, p.12,p. 3011-3020.

Cruz, Sandra A., Oliveira, Éder C., Oliveira, Fernando C. S. de, Garcia, Pâmela S., Kaneko, Manuela L. Q. A. (2011). Polímeros reciclados para contato com alimentos. Polímeros, v.21, n.4, p.340-345.

Ferment, G., Fernandes, G., Avanci, J. (2010). Seminário sobre proteção da agrobiodiversidade e direito dos agricultores: Propostas para enfrentar a contaminação transgênica do milho. Brasília: MDA.

Food And Agriculture Organization Of The United Nations. International Scientific Symposium Biodiversity And Sustainable Diets United Against Hunger. Rome, 2010. Available from:http://www.fao.org/ag/humannutrition/2850618960efe4aed57af34e2dbb8dc578d465df8b .pdf

Food and Agriculture Organization of the United Nations (FAO). THE STATE OF FOOD AND AGRICULTURE. CLIMATE CHANGE, AGRICULTURE AND FOOD SECURITY. 2016.

Geraldo, A. P. G., Bandoni, D. H., Jaime, P. C. (2008). Aspectos dietéticos das refeições oferecidas por empresas participantes do Programa de Alimentação do Trabalhador na Cidade de São Paulo, Brasil. Rev Panam Salud Publica, São Paulo, v.23, n.1, p.19-25.

Gorgulho, B. M., Lipi, M., Marchioni, D. M. L. (2011). Qualidade nutricional das refeições servidas em uma unidade de alimentação e nutrição de uma indústria da região metropolitana de São Paulo. Revista de Nutrição, Campinas, v.24, n.3, p.463-472.

Gouveia, N. (2012). Resíduos sólidos urbanos: impactos socioambientais e perspectiva de manejo sustentável com inclusão. Ciência e Saúde Coletiva, v.17, n.6, p.1503-1510.

Hall, K. D., Guo, J.; Dore, M., Chow, C. C. (2009). The Progressive increase of food waste in America and its environmental impact. Plos One, USA, v.4, n.11, e7940, 2009.

Harmon, A. H., Gerald, B. L. (2007). Position of the American dietetic association: food and nutrition professionals can implement practices to conserve natural resources and support ecological sustainability. Journal of the American Dietetic Association, United States, v.107, n.6, p.10331043.

Heller MCaK, G. A. (2015). Greenhouse Gas Emission Estimates of U.S. Dietary Choices and 1920 Food Loss. Journal of Industrial Ecology. v.19, n.3, p. 391-401.

Karam, K. M., Barboza, L. M. V. (2010). Estudo de hábitos alimentares na educação de jovens e adultos. Portal da Secretaria da Educação do Paraná, 2010. Disponível em: <www.diaadiaeducacao.pr.gov.br/portals/pde/arquivos/968-4.pdf>. Acesso em: 26 de out. 2016.

Krause, R. W., Bahls, A. A. D. S. M. (2013). Orientações gerais para uma gastronomia sustentável. Revista Turismo Visão e Ação, v.15, n.3, p.434-450.

Lamberts, R., Triana, M. A., Fossati, M., Batista, J. O. (2008). Sustentabilidade nas edificações: contexto internacional e algumas referências brasileiras na área. Laboratório de Eficiência Energética, UFSC.

Lima, T. C. (2012). Sustentabilidade em Unidades de Alimentação: Estudo sobre um Restaurante de Cozinha Brasileira. Trabalho de projeto apresentado à Escola Superior de Hotelaria e Turismo do Estoril para a obtenção do grau de Mestre em Segurança e Qualidade Alimentar em Restauração. Estoril, Janeiro de 2012. 
Lima, P. F. C., Lima, A. M. M., Castro, S. M. V., Gomes, M. V. C. N. (2015). O consumo de alimentos orgânicos na cidade de Manaus (AM): o comércio de produtos e a sustentabilidade do setor. Revista Verde, v.10, n.1, p.120-127.

MARTINS, A. M. (2015). Sustentabilidade ambiental em unidades de alimentação e nutrição coletivas de Santa Catarina. Dissertação submetida ao programa de Pós-Graduação em Nutrição da Universidade Federal de Santa Catarina para a obtenção do Grau de Mestre em Nutrição. Florianópolis, 2015.

MILLEN, B. et al. (2015). Food Sustainability and Safety. In: 2015-2020 Dietary Guidelines for Americans. Disponível em: https://health.gov/dietaryguidelines/2015/resources/20152020_Dietary_Guidelines.pdf

MORETTI, C. L. Aproveitamento de resíduos do processamento mínimo de frutas e hortaliças. São Paulo: EMBRAPA, 2006.

O'KANE, G. (2011). What is the real cost of our food? Implications for the environment, society and public health nutrition. Public Health Nutrition, v.15, n.2, p.268-276.

Real, L. C. V., Schneider, S. (2011). O uso de programas públicos de alimentação na reaproximação do pequeno produtor com o consumidor: o caso do programa de alimentação escolar. Estudo \& debate, v.18, n.2, p.57-79.

Santos, P. M. P. P., Santos, A. (2012). Produção de alimentos com responsabilidade socioambiental em Unidade de Alimentação e Nutrição. Nutrição em pauta, v.2, n.6, p. 1-5.

Santos, E. S. B., Silva, E. M. R. (2006). Estratégias vivenciadas sobre educação ambiental por professores de 1a à 4ạ série do Ensino Fundamental I. 2006. 69 f. Monografia (Especialização em Ciências Ambientais), Fundação de Ensino Superior de Olinda, Olinda, 2006.

SESI. SERVIÇO SOCIAL DA INDÚSTRIA. Cozinha Brasil. Disponível em: < http://www.sesirs.org.br/projetos_sesi.asp >. Acesso em: 19 maio 2013.

Silva, C. A., Andreoli, C. V. (2010). Compostagem como alternativa a disposição final dos resíduos sólidos gerados na CEASA Curitiba/PR. Engenharia Ambiental, v.7, n.2, p. 27-40.

Rigotto, R. M., Carneiro, F. F., Marinho, A. M. C. P., Rocha, M. M., Ferreira, M. J. M., Pessoa, V. M., Teixeira, A. C. A., Silva, M. L. V., Braga, L. Q. V., Teixeira, M. M. (2012). O verde da economia no campo: desafios à pesquisa e às políticas públicas para a promoção da saúde no avanço da modernização agrícola. Ciência e Saúde Coletiva, v.17, n.6, p.1533-1542.

Souza, A. A., Azevedo, E., Lima, E. E., Silva, A. P. F. (2012). Alimentos orgânicos e saúde humana: estudo sobre as controvérsias. Rev Panam Salud Publica, v.31, n.6, p.513-7.

Souza, A. A., Silva, A. P. F., Azevedo, E., Ramos, M. O. (2015). Cardápios e sustentabilidade: ensaio sobre as diretrizes do Programa Nacional de Alimentação Escolar. Rev. Nutr., v.28, n.2, p.217229.

TADDEI, J. A. DE A. C. et al. Nutrição em Saúde Pública. Rio de Janeiro: Rubio, 2011.

Tatàno, F., Caramiello, C., Paolini, T., \& Tripoloni, L. (2017). Generation and collection of restaurant waste: Characterization and evaluation at a case study in Italy. Waste Management, v.61, p. 423442.

Tilman, D., Clarck, M. (2014). Global diets link environmental sustainability and human health. Nature. 2014. PMID: 25383533. http://www.ncbi.nlm.nih.gov/pubmed/25383533.

Triches, R. M.; Froehlich, E.; Schineider, S. (2011). Relações de produção e consumo: a aquisição de produtos da agricultura familiar para o Programa de Alimentação Escolar no município de Dois Irmãos (RS). Rio Grande do Sul, 2011.

Veiros, M. B.; Proença, R. P. C. (2010). Princípios de sustentabilidade na produção de refeições. Nutrição em Pauta, São Paulo, p. 45-49. 
Veiros, M. B.; Proença, R. P. C.; Kent-Smith, L.; Hering, B.; Sousa, A. A. (2006). How to analyse and develop healthy menus in foodservice. Journal of Foodservice, v.17, p.159-165.

UNITED NATIONS ENVIRONMENT PROGRAMME. Towards a green economy: pathways to sustainable development and poverty eradication. S. I.: Unep, 2011.

WALLACE, A. (2005). Creating a sustainable restaurant industry with the green restaurant association. 2005. 28f. Trabalho de conclusão de estágio- Environmental Systems/Earth Science, University of California, San Diego, 2005.

WANJEK, C. (2005). Food at work: workplace solutions for malnutrition, obesity and chronic diseases. Geneva: International Labour Office, 2005.

Shcholnik, W. (2011). Qualidade, indicadores e sustentabilidade. J Bras Patol Med Lab, v.47, n3, p.198-199.

WHO. World Health Organization. WHA57.17. 57ạ Assembleia Mundial de Saúde. Estratégia Global em Alimentação Saudável, Atividade Física e Saúde. Oitava sessão plenária, 22 de maio de 2004. The World Oral Health Report, 2003a.

Zanoni, M., Ferment, G. (2011). Transgênicos para quem? Agricultura, Ciência e Sociedade. Brasília, MDA, 2011. 